\title{
EchoGéo
}

$7 \mid 2008$

Les nouveaux enjeux régionaux dans l'océan Indien occidental

\section{Les paradoxes du littoral Swahili}

Fragmentation et ouverture, entre péninsules, môles et archipels

\section{François Bart}

\section{(2) OpenEdition \\ Journals}

Édition électronique

URL : https://journals.openedition.org/echogeo/8623

DOI : 10.4000/echogeo.8623

ISSN : 1963-1197

Éditeur

Pôle de recherche pour l'organisation et la diffusion de l'information géographique (CNRS UMR 8586)

\section{Référence électronique}

François Bart, «Les paradoxes du littoral Swahili », EchoGéo [En ligne], 7 | 2008, mis en ligne le 17 octobre 2008, consulté le 01 août 2021. URL : http://journals.openedition.org/echogeo/8623 ; DOI : https://doi.org/10.4000/echogeo.8623

\section{Ce document a été généré automatiquement le 1 août 2021}

EchoGéo est mis à disposition selon les termes de la licence Creative Commons Attribution - Pas d'Utilisation Commerciale - Pas de Modification 4.0 International (CC BY-NC-ND) 


\title{
Les paradoxes du littoral Swahili
}

\author{
Fragmentation et ouverture, entre péninsules, môles et archipels
}

\section{François Bart}

1 La façade océanique de l'Afrique orientale, des côtes somaliennes au nord, jusqu'à celles du Mozambique au sud, plus précisément des alentours de Mogadiscio ( $2^{\circ}$ lat. N.) à l'embouchure du Zambèze au Mozambique ( $18^{\circ}$ lat. S.), s'allongent sur plus de 2000 kilomètres, répartis dans quatre pays, Somalie, Kenya, Tanzanie et Mozambique. Ces rivages africains de l'Océan Indien occidental, qui sont tout autant "filtre et interface » (Raison, 1994, p. 269), doivent leur identité géographique contemporaine à la conjonction de deux données essentielles: une situation privilégiée d'interface Afrique/Asie/archipels du sud-ouest de l'Océan Indien; une longue histoire de flux maritimes qui, à travers l'océan, au gré de l'alternance saisonnière des moussons, ont transporté, depuis deux millénaires, des hommes, des cultures, des produits. Cette intense vie de relations, inscrite dans la durée, vectrice de l'islam, a forgé peu à peu une aire culturelle littorale et insulaire, communément appelée aire swahili, faite de métissages, d'échanges et d'ouverture ancienne sur le monde non africain, tout particulièrement asiatique. À l'époque coloniale, les logiques de pénétration européenne vers l'intérieur du continent africain ont encore enrichi ce dispositif spatial : ce littoral est vraiment devenu un littoral à double face, façade occidentale de l'Océan Indien, façade orientale du continent africain (fig. 1). L'aire swahili a ainsi connu une nouvelle phase d'expansion, marquée par un enracinement continental croissant, conduit par les axes pénétrants, jusqu'aux confins de la Zambie et à la province congolaise du Shaba (Lubumbashi), bien au-delà du lac Tanganyika. Ce littoral est devenu une interface en situation centrale, dont le rôle classique de contact terremer est enrichi par une fonction de rencontre Afrique-Asie, s'appuyant sur quelques relais insulaires et archipélagiques (Zanzibar, Comores, Madagascar, Mascareignes...). 
Figure 1 - le littoral swahili dans son environnement maritime continental

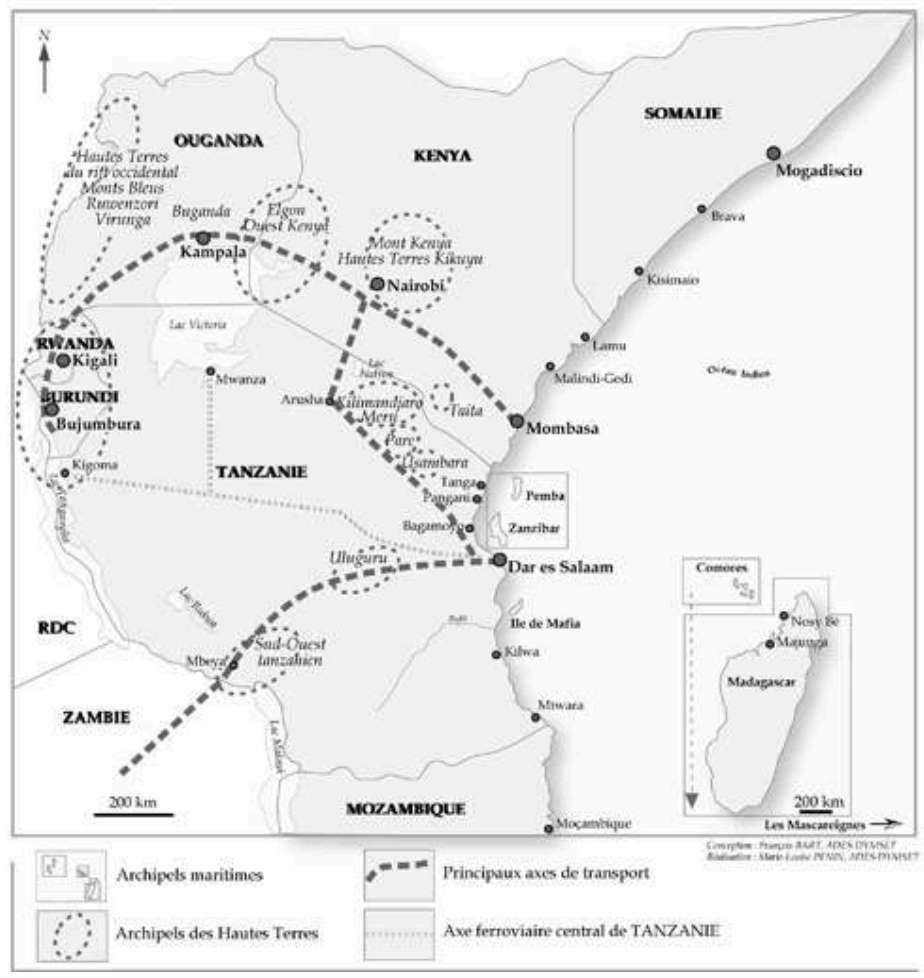

Source : F. Bart.

2 Dans le même temps, cette même histoire qui a fortement suscité l'ouverture et l'échange, a aussi, en raison de contraintes géographiques et géopolitiques, alimenté des processus de fragmentation. Dans cette aire géographique charnière, le développement, porté par les flux et les échanges, est aussi bridé par des discontinuités spatiales majeures, enracinées dans l'histoire, précoloniale et/ou contemporaine, dans une géographie du morcellement et de la longue distance. On a là un jeu complexe où s'imbriquent des dynamiques d'ouverture et d'isolement, de fusion et de fragmentation, de blocage et de transgression. Et dans cette aire très vaste, à la fois maritime et continentale, dont le littoral serait le centre et dont Zanzibar aurait pu, dans une logique de "légitimité » historique, être l'épicentre, ce jeu de forces contraires concerne tout autant le versant continental que le versant maritime : à celui-ci les archipels, les îles, les aléas de navigation des boutres et des cargos, à celui-là le morcellement induit par un dispositif de môles très dispersés de hautes terres, véritables noyaux de développement, séparés les uns des autres par des immensités peu peuplées, volontiers sèches, traditionnellement jugées répulsives.

\section{Continuités et discontinuités de l'hinterland continental}

3 La façade océanique est-africaine est le poumon d'un vaste ensemble continental, l'Afrique des Rifts, marqué par une organisation spatiale méridienne, par la présence de quelques-uns des plus grands lacs du monde (Lacs Victoria, Tanganyika, Malawi) et par l'étendue des terres d'altitude. 


\section{Les môles de hautes terres densément peuplées et cultivées}

Dispersés parmi ces vastes étendues continentales, plusieurs ensembles de hautes terres (massifs montagneux, hauts plateaux, collines d'altitude élevée) structurent l'organisation de cet arrière-pays du littoral swahili : les plus significatifs se trouvent :

- dans le quart sud-ouest du Kenya, du mont Kenya au mont Elgon et aux rives du lac Victoria,

- tout au long de l'arc montagneux tanzanien, des monts Meru et Kilimandjaro au nord aux monts Rungwe et Poroto au sud-ouest, en passant par les monts Pare, Usambara et Uluguru,

- en Ouganda méridional et occidental, depuis les collines du Buganda, proches du lac Victoria, lui-même à plus de 1100 mètres d'altitude, jusqu'aux très hautes cimes du Ruwenzori, sur la frontière du Congo et des monts Virunga, partagés avec le Rwanda,

- la quasi-totalité de la surface du Rwanda et du Burundi,

- quelques massifs de la frange orientale de la République Démocratique du Congo, au nordKivu (monts Bleus, mont Hoyo, régions de Beni, Lubero, Masisi).

5 Avec ces derniers ensembles, on parvient aux limites de l'Afrique centrale et de la cuvette congolaise ; néanmoins, aussi éloigné soit-on du littoral de l'Océan Indien - plus de 1500 kilomètres du port de Mombasa à la capitale du Rwanda, Kigali - on est ici, y compris dans l'est de la RDC, dans une partie du continent très dépendante du fonctionnement des communications avec les ports kenyans et tanzaniens.

Ces môles de hautes terres présentent quelques caractéristiques communes qui en font des pôles structurants :

- de très fortes densités de population, liées à des systèmes paysans agro-pastoraux: $321 \mathrm{hab} . / \mathrm{km}^{2}$ en moyenne au Rwanda (recensement de 2002), des valeurs équivalentes au Kenya en pays kikuyu, plus fortes encore dans l'ouest autour de Kisii ; on note aussi le très fort peuplement du pays Chagga sur les pentes du Kilimandjaro, de la quasi-totalité du Burundi etc.

- Des points d'ancrage pour un réseau urbain en devenir. Ces très fortes densités rurales deviennent aussi le socle de gros îlots de forte densité en continuum ville-campagne, comme au nord de Nairobi, autour d'Arusha, ou de Kampala. Si le littoral abrite quelques métropoles et grandes villes, Dar es Salaam et Mombasa surtout, l'intérieur du continent est loin d'en être dépourvu, avec Nairobi, Kampala, Arusha, Kigali, Bujumbura, et des villes secondaires en forte croissance. Ces môles de hautes terres sont le principal support spatial du rattrapage du retard de l'urbanisation est-africaine. C'est là que l'on trouve en particulier la capitale du Kenya, Nairobi, qui, à bien des égards, vu son poids économique et son rôle de hub, peut être considérée comme la métropole de cette partie du continent.

- Des systèmes agricoles élaborés, favorisés par de bonnes aptitudes pédologiques et climatiques, qui, à la fois, fournissent une large gamme de produits vivriers (maïs, haricots, légumes, fruits, etc.) et procurent des exportations constituant autant de piliers pour les économies nationales : le café et le thé dans tous les pays, auxquels il faut ajouter les fleurs et certains légumes (surtout au Kenya).

- Des espaces touristiques de renommée mondiale. Si le littoral, surtout au Kenya et dans l'archipel de Zanzibar, a connu un boom touristique important, l'histoire du tourisme estafricain a commencé par l'intérieur, autour des réserves de chasse et des grands parcs nationaux. Certains môles de hautes terres sont devenus des espaces touristiques majeurs: en Tanzanie, le mont Kilimandjaro a même, sur son piémont, son propre aéroport international, directement relié à l'Europe; le massif du Ngorongoro est l'une des plus 
fameuses réserves animalières du monde, dans et autour d'une immense caldeira; le mont Kenya au Kenya, le Ruwenzori en Ouganda, les monts Virunga, célèbres pour leurs gorilles de montagne, au Rwanda, constituent aussi des espaces très attractifs quand la sécurité des visiteurs est assurée.

Dans l'intérieur du continent, le grand tourisme de nature n'est pas cantonné aux môles de hautes terres; il concerne aussi des espaces plus secs, qui abritent des parcs aussi célèbres que le Serengeti, le Masaï Mara, Amboseli et le Tsavo. La valorisation économique de ces espaces, peu peuplés, ou vides, renforce le poids de l'hinterland dans une activité touristique devenue, au Kenya et en Tanzanie, un moteur important du développement.

\section{Vides et césures}

7 La géographie de ces môles de hautes terres, qu'ils soient des volcans (Kilimandjaro, Meru, Ngorongoro, Kenya, Elgon, Virunga, Ringwe...) ou des horsts (Ruwenzori, Usambara, Crête Congo-Nil...), se caractérise d'abord par leur dispersion: aucun ensemble montagneux continu, mais de grandes étendues d'espaces plus bas, plus secs, peu peuplés, qui les séparent. Ces terres de pori (brousse, bush) ont suscité longtemps la crainte des voyageurs et restent pour les populations montagnardes peu attractives quand bien même certaines d'entre elles servent de déversoirs démographiques aux excédents de population des massifs.

8 La géographie continentale est-africaine est ainsi une géographie d'archipels montagnards dont chaque composante a une forte identité ; de surcroît, ces massifs, qui concentrent les terres agricoles les plus fertiles, celles d'un étage moyen (1500-2 000 mètres) jouissant souvent de sols fertiles et d'un indéniable privilège pluviométrique et sanitaire, sont souvent des îlots de relative richesse et modernité. Entre eux, les vides constituent des espaces interstitiels, voire de véritables césures/ fractures, pour peu que l'insécurité y sévisse comme dans le nord du Kenya et de l'Ouganda. L'organisation spatiale de la Tanzanie, où toutes les régions les plus actives sont en situation géographique de périphérie - littorale (Dar es Salaam), et intérieure montagnarde (Kilimandjaro, Arusha, Mbeya) - est tout à fait caractéristique de cette géographie de fragmentation, autour d'un centre vide et mou («tache aveugle», Raison, 1994, p. 346) que la création de la nouvelle capitale politique, Dodoma, n'a guère modifiée.

Dans ce contexte d'éparpillement, la géographie des principaux axes de communication va contribuer à projeter sur le littoral de l'Océan Indien (à moins que ce ne soit l'inverse) cet éclatement spatial.

\section{Les axes reliant l'intérieur au littoral}

Ils s'inscrivent tous, de façon très classique, dans la logique de la pénétration, celle des anciennes routes caravanières des marchands arabes pratiquant le commerce de l'ivoire et des esclaves, celle ensuite héritée de la colonisation, allemande puis britannique essentiellement. Tous aussi, de par la configuration géographique précédemment décrite, doivent s'affranchir de longues distances d'étendues de savanes et de forêts sèches (miombo), séparant la frange littorale des bastions montagnards intérieurs. 
11 La dispersion des composantes de l'archipel des hautes terres intérieures est ainsi venue renforcer celle des comptoirs littoraux, même si l'évolution contemporaine se caractérise ici, comme partout, par une tendance à la concentration des équipements et des infrastructures.

D'une façon schématique, on peut esquisser trois principales «projections » de l'archipel continental sur le linéaire côtier : au sud, des hautes terres de la région de Mbeya vers Dar es Salaam ; au centre, d'Arusha et du Kilimandjaro vers le port de Tanga ; au nord, des hautes terres du Burundi, du Rwanda, d'Ouganda et du Kenya vers le port de Mombasa. Dans les faits, l'histoire coloniale et des évolutions géopolitiques ou géo-économiques contemporaines ont fait évoluer la donne, en concentrant les flux sur deux débouchés littoraux :

- Dar es Salaam a été, dès 1910, à l'époque allemande, le point de départ d'une voie ferrée vers les lacs Tanganyika (Kigoma) et Victoria (Mwanza), à l'écart des principaux pôles d'activité des hautes terres, dans la mesure où le prolongement vers le Rwanda, évoqué depuis des décennies, n'a pas été réalisé à ce jour. Ce corridor, qui ne repose que sur une voie ferrée obsolète, ne présente guère plus qu'une valeur symbolique.

Pourtant, le rôle de Dar es Salaam s'est renforcé pour deux raisons : d'une part, le déclin du port de Tanga, qui ne conserve qu'une fonction d'étape pour le cabotage, a fait de Dar es Salaam le principal débouché de l'importante région d'Arusha-Kilimandjaro, grâce à l'asphaltage total et à l'amélioration d'un axe routier, à dimension internationale (OugandaKenya-Tanzanie), qui a complètement supplanté les lignes de chemin de fer moribondes reliant Dar es Salaam et Tanga à Moshi et Arusha. D'autre part, l'histoire politique de l'Afrique australe à l'époque de l'apartheid en Afrique du Sud a fait participer la Tanzanie socialiste du président Nyerere au front anti-apartheid; elle est devenue ainsi le débouché maritime privilégié de la Zambie et du Malawi, par l'intermédiaire de l'axe routier totalement asphalté Mbeya-Dar es Salaam (800 kilomètres), doublé depuis 1968 par un pipe line et depuis 1976 par une voie ferrée construite par les Chinois, le Tazara.

- Mombasa est la tête de pont ancienne de la voie ferrée qui, via Nairobi, dont elle a suscité la promotion au statut de capitale en 1905, a atteint Kisumu dès 1902, puis Kampala plus tard. Elle est aujourd'hui doublée par un axe entièrement asphalté reliant Mombasa à Bujumbura, sur une distance de près de 2000 kilomètres. L'hinterland du port de Mombasa s'est d'autant plus agrandi que les efforts du Burundi et du Rwanda pour diversifier leurs débouchés maritimes par la Tanzanie n'ont pas encore abouti à une modification profonde des flux, et que l'instabilité politique de la RDC et du Soudan ont contribué à orienter davantage le Kivu congolais et le sud Soudan vers la route de Mombasa.

Cet axe, doublé par un oléoduc entre Mombasa et Nairobi, est devenu le plus important de toute l'Afrique orientale et contribue à faire basculer de plus en plus vers l'est l'économie de l'Afrique des Grands Lacs.

\section{Les passerelles et ancrages océaniques}


Figure 2 - L'océan indien occidental entre Afrique, Asie et archipels

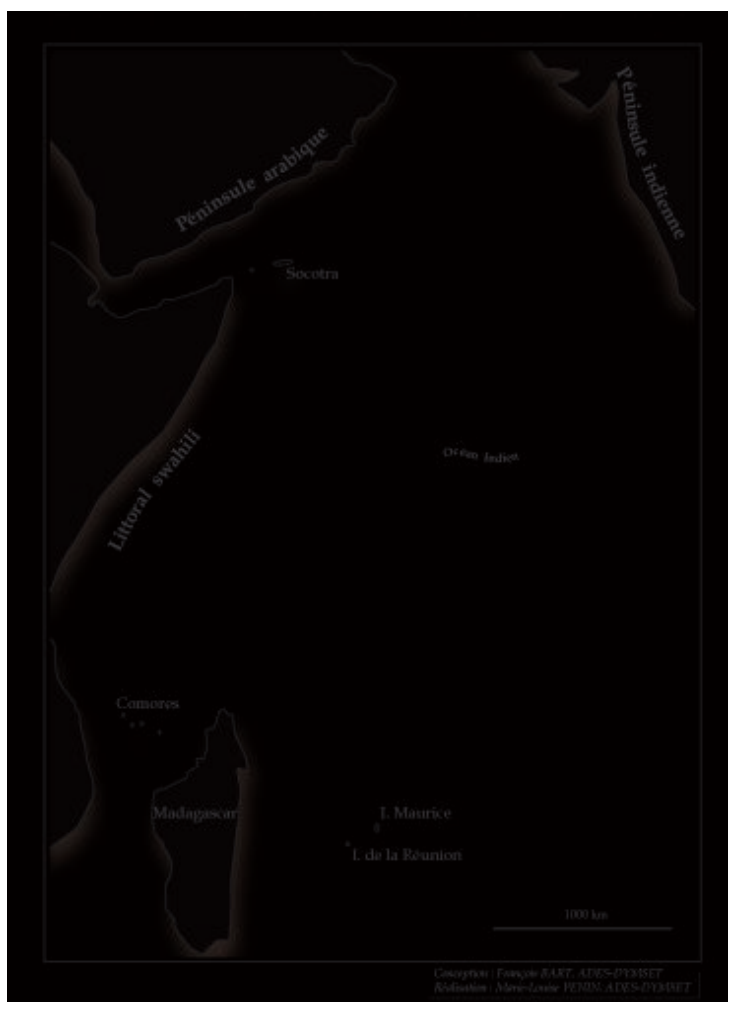

Source : F. Bart.

14 Le littoral est-africain de l'Océan Indien bénéficie de longue date d'une tradition maritime qui, par les mouvements des boutres, a résolument tourné vers l'est lointain cette partie du continent, où les comptoirs arabes ont largement précédé les installations européennes. Diverses sources, archéologiques et autres attestent en effet que :

Incursions phéniciennes, juives peut-être, grecques et perses probablement, tous les peuples entreprenants et commerçants cherchent, précautionneusement, la source de l'or, de l'encens, de l'ivoire. Pendant longtemps (700 av. J.-C.-650 apr. J.-C.) des migrations préislamiques d'Arabes, de Persans et d'Indiens établissent sur la côte des comptoirs commerciaux sans doute actifs (Bourde, 1968, p. 34).

\section{Les grandes péninsules d'Asie occidentale}

16 Au cours de cette longue histoire, au cours de laquelle des peuples aussi éloignés que les Chinois se seraient intéressés à ce littoral (cf. vestiges de porcelaines chinoises à Kunduchi près de Dar es Salaam), deux grandes péninsules asiatiques ont largement contribué à la construction de cette identité swahili, terme dont il faut rappeler la signification étymologique, à partir du mot arabe sawahili, qui signifie «le peuple côtier » (Sheriff, in Bernardie, 2008 p. 296). 


\section{La Péninsule arabique}

17 C'est par là que sont arrivés des marchands diffusant leur religion, l'islam, le vocabulaire arabe (l'une des composantes de la langue syncrétique qu'est le swahili), des pratiques sociales et culturelles spécifiques.

18 Les Arabes, tout particulièrement les Yéménites et les Omanais, ont été, aux côtés des Portugais, les grands bâtisseurs de comptoirs, de Mogadiscio à Sofala : on peut citer, en Somalie, Brava et Kisimaio, au Kenya, Lamu, Malindi et Mombasa, en Tanzanie, Zanzibar, Mafia et Kilwa, au Mozambique, Moçambique et Quelimane. Dans la partie sud, les Portugais ont joué un rôle important; installés à Sofala dès 1525, ils ont aussi occupé Quelimane, Moçambique et, moins durablement, Kilwa et Mombasa, où l'on visite encore le Fort Jesus, à la lisière d'une vieille ville qui ressemble beaucoup à une médina.

19 L'apogée de l'influence politique d'acteurs issus de la péninsule arabique se situe au $\mathrm{XIX}^{\mathrm{e}}$ siècle, quand se constitue sur ce littoral un empire « omano-zanzibari », à vocation essentiellement commerciale (girofle, ivoire, esclaves), dirigé par les sultans d'Oman, qui installent leur capitale... à Zanzibar en 1832 : exemple rare d'une " délocalisation " politique d'un continent à l'autre, qui va fortement marquer la vie d'une partie de ce littoral, de Zanzibar à Mombasa, jusqu'à ce que les Britanniques l'incorporent à leur empire à partir de 1890 .

Si la révolution de Zanzibar en 1964 a particulièrement visé (et chassé) cette population arabe, la question des liens du littoral swahili avec la Péninsule arabique, et plus largement le monde musulman, se pose aujourd'hui en deux registres principaux :

- l'émergence d'un mouvement islamique radical (ansar as-sunna) (Loimeier in Bernardie, 2008 p. 328 ), qui s'inscrit à la fois dans des dynamiques de tensions politiques locales et internationales et qui peut apparaître sur ce littoral comme un nouveau faciès de l'antagonisme ancien entre « islam africain » (celui des confréries soufies) et « islamoarabe » (Constantin 1987).

- L'intensification spectaculaire des flux d'hommes et de marchandises entre la côte swahili et les pays du Golfe, tout particulièrement Dubai. Le trafic traditionnel de cabotage de marchandises diverses assuré par des petits cargos et des boutres entre les ports arabiques et les ports swahili reste significatif ; on assiste néanmoins à un renouveau de cette vie de relations.

21 D'une part, la multiplication des liaisons aériennes directes entre les aéroports littoraux (Dar es Salaam, Zanzibar et Mombasa) et le Golfe Persique a renforcé des flux d'émigration de travail de Swahili vers le Golfe. Dar es Salaam avait par exemple 15 liaisons hebdomadaires en 2005 avec la Péninsule arabique, presque autant qu'avec l'Europe (Calas, 2006, p. 322). Des enquêtes menées chez des fermiers de l'archipel zanzibari en juin 2006 nous ont montré à quel point l'argent du Golfe est devenu une composante essentielle de la vie de bien des ménages, comme si Dubai devenait la véritable capitale économique de l'archipel. De plus, la diaspora zanzibari a dans cette Péninsule arabique l'un de ses points d'ancrage fort (Saleh M., in Bernardie, 2008, p. 278-279), intéressant renversement de l'histoire... Enfin le contexte de relative libéralisation économique fait aujourd'hui des ports swahili d'importants relais, non seulement du trafic de pétrole, mais aussi de produits manufacturés des pays du Golfe (électronique, électro-ménager...). 


\section{La Péninsule indienne} Kenya, puis le Tanganyika des pages de l'histoire de l'empire colonial britannique. De 1834 à 1907, 450000 Indiens y arrivèrent, 300000 d'entre eux firent souche, et les Indiens y détenaient en $192145 \%$ des surfaces de canne à sucre (Raison, 1994 p. 447). Elle a ainsi participé au processus global d'« indianisation » de cette partie de l'océan Indien, qui contribue donc à rapprocher littoral swahili et Mascareignes. Aujourd'hui le dynamisme économique de sociétés à capitaux indo-mauriciens est présent en Afrique de l'Est dans les secteurs du sucre et du tourisme; Maurice est devenu l'un des plus gros investisseurs étrangers au Mozambique.

D'autre part, l'île de la Réunion a été un poste avancé de la pénétration européenne vers le littoral swahili. C'est de cette île que sont partis vers Zanzibar en 1860 les premiers missionnaires catholiques de la Congrégation du Saint Esprit ; c'est à partir de cette île aussi que sont partis, sans doute en 1877, les premiers plants de caféiers vers l'Afrique de l'Est, dont des graines auraient été apportées de la Réunion par des missionnaires spiritains, dont le père Horner ; la présence du caféier, introduit par les Arabes, à Zanzibar est en effet attestée par une description de 1866 contenue dans le diaire de la mission Saint Joseph; cette culture, dont on connaît l'importance 
économique actuelle dans les hautes terres a ensuite été diffusée dans l'intérieur à partir de Zanzibar et Bagamoyo (Lenoble-Bart 2002).

Ces deux îles doivent enfin l'essentiel de leur peuplement de souche africaine, descendants d'esclaves, souvent appelés Cafres, aux flux qui, via les ports swahili, et sous la houlette des sultans de Zanzibar, ont amené vers les îles des individus issus en particulier du Mozambique, mais aussi de l'intérieur du continent.

C'est enfin la diffusion de l'islam qui semble avoir été le support des relations anciennes entre la côte nord-ouest de Madagascar, l'archipel comorien, «situé comme un pont entre le continent africain et le monde des îles» (Raison, 1994, p. 438) et la côte swahili. La société comorienne s'est constituée au gré d'innombrables flux qui, du Xe au XVI ${ }^{e}$ siècles sont venus "de Chiraz, d'Oman, de Mascate, du Yemen, de Zanzibar et de Pate" (ibid. p. 439) et, pendant toute la période coloniale, les Comores ont été un foyer d'émigration d'un côté vers Zanzibar et le Tanganyika, de l'autre vers Madagascar. La civilisation swahili, usant du relais comorien, poussa ainsi des antennes jusqu'au littoral malgache de Majunga. Comme l'écrit Marie-Pierre Ballarin (Bernardie-Tahir, 2008, p. 238), Madagascar, Comores et Zanzibar ont une " histoire commune méconnue », l'île de Nosy Bé ayant été "l'île-carrefour de la zone » (ibid.).

Entre Comores et Zanzibar existe ainsi une forte complicité historique, dont l'un des résultats tangibles est la parenté des langues comoriennes et du swahili. Dans cette logique de passerelle, l'archipel de Zanzibar, aux portes du littoral africain, a joué un rôle essentiel.

\section{Le littoral swahili : atouts et contraintes de développement}

31 Le littoral swahili, dans une première approche, présente une indéniable unité (Calas 2003), bien au-delà de l'héritage historique, religieux et humain, qui constitue le ciment identitaire fondamental. Quelques autres éléments majeurs peuvent être énoncés :

- il constitue une étroite frange littorale densément peuplée, séparée des bastions ethnodémographiques intérieurs par des étendues au peuplement très lâche ; cette configuration contribue fortement à la spécificité de l'identité littorale. Entre Malindi et le delta de la Rufiji, et à l'extrême sud au niveau de Mtwara, les densités atteignent $100 \mathrm{hab} . / \mathrm{km} 2$ (350 hab. $/ \mathrm{km}^{2}$ dans les îles), alors que les solitudes continentales proches se contentent de moins de $12 \mathrm{hab} . / \mathrm{km}^{2}$. Cette répartition de la population s'explique à la fois par le pouvoir attractif des ressources de la mer et par le fort gradient pluviométrique existant entre le trait de côte (1200 mm à Mombasa) et l'intérieur proche (à peine $700 \mathrm{~mm}$ à 50 kilomètres de Mombasa).

- Il abrite des populations d'agriculteurs et de pêcheurs. Si la pêche, dans les lagons et en haute mer, est très inégalement répartie le long du littoral, elle est un « trait de civilisation » significatif et a ponctuellement, à Zanzibar, dans la zone Tanga-Dar es Salaam, autour de Kilwa et de Mtwara, sur le nord du littoral mozambicain, une fonction économique importante : poissons divers (thon etc.), crustacés (crevettes du Mozambique, langoustes...) sont parfois complétés par la culture des algues (Zanzibar, certaines criques de la côte kenyane).

- L'agriculture, sur les sols coralliens ou sablonneux du cordon littoral, mêle de nombreux produits vivriers, des produits maraîchers pour l'alimentation des villes et des complexes 
touristiques, une importante arboriculture fruitière associant manguiers, cocotiers et anacardiers (ces derniers surtout dans le sud de la Tanzanie ou au Mozambique. Il existe aussi des plantations de sisal (Tanga) et de girofliers (Zanzibar).

- Il est jalonné par un grand nombre de petites villes portuaires et commerciales, plus ou moins actives ou alanguies, héritage des comptoirs arabes. Certaines conservent des témoignages historiques et archéologiques intéressants, qui servent parfois de support à un tourisme à dimension culturelle. En dehors du plus fameux de ces centres, Zanzibar, dont il sera question ci-après, on peut citer, sur de petites îles proches du littoral, Lamu (Kenya), devenue " paradis touristique »), Kilwa (Tanzanie), dont les ruines en bordure de mangrove sont en cours de réhabilitation, Moçambique (Mozambique); sur le littoral même, Gedi au Kenya, Bagamoyo en Tanzanie recèlent, comme Zanzibar, Lamu et Kilwa, de précieux témoignages d'une architecture swahili (Pradines S., in Charlery de la Masselière, 1998), faite de maisons de marchands aux portes sculptées, de forts et d'enceintes, de mosquées, que ces pays pauvres tentent de restaurer. Parmi tous ces établissements littoraux, Zanzibar occupe une place particulière.

\section{Zanzibar, cœur du littoral swahili ?} migratoires, innombrables mobilités, échanges de produits bruts et manufacturés, rôle des réseaux religieux, familiaux et marchands sont là pour témoigner de cette ouverture au monde. Mais dans le même temps, Zanzibar, intégrée dans la République Unie de Tanzanie, présente de nombreux symptômes de marginalisation: politique, dans la mesure où le pouvoir, malgré un statut spécifique, est sur le continent; économique, car l'économie du girofle est en difficulté (Bart F., in Bernardie-Tahir, 2008, p. 69-84), et la dépendance alimentaire vis-à-vis du continent s'accroît; sociale, parce que la majorité de la population n'est pas sortie de la pauvreté, et se trouve contrainte de chercher sur le continent et sous des cieux lointains leurs moyens de survie ; démographique enfin, car que pèse Zanzibar par rapport à Dar es Salaam, forte d'une population dix fois plus nombreuse? Sur ce dernier point, on peut évoquer, depuis 1964 «le long déclin de Zanzibar par rapport à Dar es Salaam »(Robert, in Calas, 2006, p. 341).

En ce début du XXI ${ }^{e}$ siècle Zanzibar est devenue un nom connu partout à travers le monde, à l'égal de son "voisin», le Kilimandjaro. L'intégration de Zanzibar dans 
l'économie-monde du tourisme, si elle manifeste une forme de permanence de l'ouverture de cet archipel, ne suffira sans doute pas à le sortir de l'angle mort politique, générateur de tensions, dans lequel il est quelque peu enfoncé, ni d'une pauvreté dont les conséquences peuvent être exacerbées par les inégalités et instrumentalisées par certains.

\section{Deux grands pôles portuaires et urbains}

Parmi les nombreuses villes et bourgades littorales, deux pôles majeurs émergent nettement: Mombasa (Kenya) et Dar es Salaam (Tanzanie), qui ont respectivement 900000 et près de 3000000 habitants. Ces deux grandes villes partagent quelques grandes caractéristiques communes : une histoire enracinée dans celle des comptoirs arabes, des points de départ de la pénétration européenne vers l'intérieur du continent, de remarquables sites portuaires de rias, une activité industrialo-portuaire à l'échelle de leurs pays respectifs, mais aussi de l'ensemble de l'Afrique orientale, jusqu'aux confins de l'Afrique centrale et de l'Afrique australe. Aucune n'est capitale politique: c'est Nairobi pour le Kenya et Dodoma pour la Tanzanie. Mais dans ce dernier pays, la prépondérance de Dar es Salaam est écrasante.

Dar es Salaam : un port dans la ville (Calas, coord., 2006)

La superbe ria de Mzizima, qui s'enfonce de plus de huit kilomètres à l'intérieur des terres, déploie un vaste plan d'eau, bien abrité, large de plusieurs centaines de mètres, profond de 8 à 12 mètres (Calas \& Bart, 1999). Le port est ainsi au cœur de l'immense métropole tanzanienne, il est aussi sa raison d'être; expression sublime d'une métropolisation swahili, qui s'est déployée dans la continuité historique d'un comptoir arabe fondé en 1862 par un sultan d'Oman, Majid, auquel a succédé une ville allemande en 1887 ; elle devient capitale de la nouvelle colonie, à la place de Bagamoyo, à partir de 1891. Le port ne prend une véritable ampleur qu'à la fin de l'époque coloniale britannique, dans le cadre d'une politique de grands travaux d'équipements; il présente la particularité de s'être développé vers l'intérieur, de l'aval vers l'amont : en 1966, par exemple, la raffinerie de pétrole a été construite à plus de trois kilomètres en retrait des premières installations portuaires de l'époque allemande.

Le volume du trafic demeure modeste, mais a nettement augmenté depuis 2000 (3,8 millions de tonnes), pour atteindre 6,3 millions de tonnes en 2005 (Tanzania Ports Authority); l'industrialisation est à l'échelle de l'un des pays les plus pauvres de cette région du monde, qui connaît cependant un taux de croissance non négligeable. La concurrence est rude avec les ports mozambicains récemment réhabilités, les ports sud-africains et, plus encore le proche voisin kenyan, Mombasa.

\section{Mombasa : la ville dans son port}

40 Le vieux comptoir arabe de Mombasa, pris ensuite par les Portugais, s'était installé dans une petite île sise entre deux rias. La vieille ville occupe aujourd'hui cette île, sertie entre le vieux port des boutres au nord et le port moderne de Kilindini au sud. L'agglomération, l'aéroport international, les zones industrielles (raffinerie) se sont développés en dehors du site insulaire originel qui, à proximité de Fort Jesus, est une composante de l'espace touristique littoral. Le port, débouché principal du « corridor 
nord » dessert, au-delà de Nairobi, le Kenya, le Rwanda, le Burundi et même la frange orientale de la RDC et le sud Soudan. Il a aujourd'hui un trafic, en progression régulière, double de celui de son concurrent tanzanien, avec 12,5 millions de tonnes en 2005 (14,1 en 2006) (Kenya Ports Authority http//www.kpa.co.ke) : il est sans conteste le plus actif de ce littoral swahili.

41 À la différence de Dar es Salaam, Mombasa est aussi au centre d'un important espace touristique, qui s'étend, sur environ 200 kilomètres de côte, de Malindi au nord, à la frontière tanzanienne au sud. Il s'agit du plus important littoral balnéaire d'Afrique tropicale, en termes d'équipements et de fréquentation. Dans le système touristique national, les attraits du littoral swahili sont souvent proposés en complément des richesses naturelles de l'intérieur, comme, de l'autre côté de la frontière, le couple bien rodé Zanzibar-Kilimandjaro.

\section{Césures littorales : un espace fragmenté}

42 Certains indicateurs, tous antérieurs à la crise kenyane de janvier 2008, montrent un indéniable dynamisme de ce littoral swahili. Pourtant, il reste profondément marqué par une fragmentation spatiale produite par d'importantes césures, qui introduisent des ruptures spectaculaires dans les systèmes de communication. Plusieurs types de facteurs interviennent: certains sont d'ordre naturel, comme des zones deltaïques marécageuses, des cours d'eau, des bras de mer difficiles à traverser; on peut citer la basse vallée de la Tana entre Malindi et Lamu, les rias de Kilifi et du sud de Mombasa (bacs), le littoral nord-tanzanien entre Pangani et Bagamoyo, la basse vallée de la Rufiji, et le fleuve Ruvuma, sur la frontière Tanzanie-Mozambique. Les progrès des infrastructures de transports permettent de s'affranchir toujours un peu mieux de ces obstacles naturels.

Plus contraignants sont les effets-frontières, et, éventuellement, les zones d'insécurité qui les bordent. Le cas le plus flagrant concerne la zone frontalière Kenya-Somalie, qui rend problématique et dangereuse la liaison Malindi-Kisimaio, au point d'obliger la plupart des touristes à se rendre à Lamu en avion. Ajoutons que la Somalie, en guerre depuis 17 ans, est bordée par l'étendue maritime la plus dangereuse du monde : 31 attaques de pirates en 2007, plus que dans la région du détroit de Malacca (FR3, Thalassa, 29 février 2008). Si la tradition de piraterie maritime semble ancienne sur cette frange nord du littoral swahili, on peut se demander si l'accroissement d'une insécurité alimentée par le chaos somalien n'est pas susceptible de gravement compromettre les flux reliant Afrique et Péninsule arabique. D'autres angles morts spectaculaires subsistent ; le plus étonnant, au centre du littoral swahili, se traduit par le mauvais état de l'axe routier qui, de Tanga, devrait acheminer les flux entre Kenya et Tanzanie; on a là peut-être un héritage du glacis qui a longtemps séparé l'empire britannique des possessions allemandes. De même, la relation Dar es SalaamMozambique, très longue (700 kilomètres de la métropole tanzanienne jusqu'à la frontière) reste problématique, malgré les améliorations récentes de cet axe, par lequel aucun pont ne permet de traverser le fleuve-frontière.

Ces ruptures spatiales rappellent que ce littoral très ouvert sur l'extérieur n'en a pas fini avec des logiques d'enclavement ponctuel qui nuisent à un fonctionnement cohérent de cette façade africaine de l'Océan Indien. 

mosaïque, un littoral globalement fragile. En effet, frange de rencontre de cultures, il suscite de nombreux appétits, que ce soit auprès d'acteurs locaux que d'acteurs du système-monde, dans le registre du politique, de l'économique, et des enjeux environnementaux.

Les longues étendues d'un linéaire côtier aux paysages emblématiques, comme les surfaces restreintes et souvent très densément peuplées des petites îles bordières, voient de plus en plus entrer en compétition des acteurs aux desseins divers, qui ont tous en commun la quête du profit et / ou du pouvoir. La pauvreté d'une majorité de la population, qui côtoie des îlots de richesse discrets ou affichés, accroît les risques de tensions sociales et politiques, sur fond d'incertitudes foncières et d'inquiétudes quant au lendemain. La diversité des héritages coloniaux et post-coloniaux, le chaos somalien, les troubles du Kenya, les incertitudes socio-politiques relatives à l'avenir de Zanzibar rappellent que la beauté apaisante des paysages n'est parfois qu'un leurre, alors même que l'appartenance à une aire culturelle swahili dont les limites s'enfoncent à présent loin à l'intérieur du continent pourrait être un gage de stabilité.

\section{BIBLIOGRAPHIE}

BAROIN C. \& CONSTANTIN F. (dir.), 1999. La Tanzanie contemporaine. Paris-Nairobi, Karthala-IFRA.

BERNARDIE-TAHIR N., 2000. Zanzibar, entre africanité et insularité. Acta Geographica, n 124 , p. 20-38.

BERNARDIE-TAHIR N. (dir.), 2008. L'autre Zanzibar. Pessac-Limoges-Paris, ADES/DYMSET-GEOLABKarthala, 380 p.

BOURDE A., 1968. L'Afrique orientale. Paris, Presses Universitaires de France, 128 p. (Que sais-je ? n 1308).

CALAS B., 2003. chap. 8 et 12, In : Bart F. (dir.), L'Afrique, continent pluriel, Paris, CNED-SEDES, 256 p.

CALAS B. (coord.), 2006. De Dar es Salaam à Bongoland. Pessac-Nairobi-Paris, ADES/DYMSET-IFRAKarthala, 390 p.

CALAS B. \& BART F., 1999. Dar es Salaam : du port au territoire swahili, in Mainet G. dir., îles et littoraux tropicaux, Actes des VIIes journées de géographie tropicale, Brest, Ouest Éditions Presses Académiques, Brest, p. 333-345.

CHARLERY DE LA MASSELIÈRE B. (dir.), 1998. Études sur la côte de l'Océan Indien. Nairobi, IFRA Les Cahiers, $76 \mathrm{p}$.

CONSTANTIN F., 1987. Les voies de l'islam en Afrique orientale. Paris, Karthala, 150 p.

CONSTANTIN F., 1991. «Fin de races ou fin de classe ? Inégalités, représentations et pouvoir sur la côte swahili est-africaine ", Annuaire des pays de l'Océan Indien, XI, CNRS \& PUAM, p. 43-64.

De VERE ALLEN J., 1993. Swahili Origins. Eastern African Studies, James Currey, Londres, 272 p. 
GRIGNON F. \& PRUNIER G. (dir.), 1998. Le Kenya contemporain. Paris-Nairobi, Karthala-IFRA

GUEBOURG J.-L., 1999. Petites îles et archipels de l'océan Indien. Paris, Karthala, 570 p.

HOORWEG J., FOEKEN D. et OBUDHO A.A. (eds.), 2000. Kenya Coast Handbook Culture, resources and development in the East African littoral. LIT, African Studies Centre, Leiden, 527 p.

HOYLE B.S., 1997. Port, port cities and coastal zones : development, interdependence and competition in East Africa. Académie Royale des sciences, t. 19, fasc. 2, Bruxelles, 67 p.

HOYLE B.S. \& HILLING D. (eds), 1997. Seaports and development in tropical Africa. Macmillan, Londres, p. 225-245

Le COUR GRANDMAISON C. et CROZON A. (dir.), 1998. Zanzibar contemporain. Karthala-IFRA, ParisNairobi, $378 \mathrm{p}$.

Le GUENNEC COPPENS F. \& CAPLAN P. (dir.), 1991. Les Swahili entre Afrique et Arabie. Karthala, Paris, $215 \mathrm{p}$.

LENOBLE-BART A., 2002. Innovations agricoles missionnaires en Afrique de l'Est, in : Routhier G. \& Laugrand F. (dir.), L'espace missionnaire. Lieu d'innovation et de rencontres interculturelles, Paris, Karthala, p. 373-393

RAISON J.-P., 1994. 5e, 6e et 7e parties, in ; Dubresson A. et al., Les Afriques au sud du Sahara, ParisMontpellier, Belin-Reclus (Géographie Universelle), $480 \mathrm{p}$.

ROY C., 2006. Une ville du Sud dans la mondialisation: Dar es Salaam et le système Monde. Thèse géographie, Pessac, Université Michel de Montaigne Bordeaux 3, 413 p.

RICHMOND M. D. (ed.), 1996. A Guide to the Seashores of Eastern Africa and the Western indian Ocean Islands. Sida, SAREC, Stockholm, $250 \mathrm{p}$.

SHERIFF A. (dir.), 1995. The history and conservation of Zanzibar Stonetown, EAS. James Currey, Londres, $151 \mathrm{p}$.

SHERIFF A., 1987. Slaves, spices and ivory in Zanziba. James Currey, Londres, , 297 p.

\section{RÉSUMÉS}

Le contact entre l'océan Indien occidental et le continent africain a forgé l'identité et l'originalité $\mathrm{du}$ littoral swahili. Cette interface, longue d'environ 2000 kilomètres, du sud de la Somalie au nord du Mozambique, constitue un monde original où, au gré de traditions anciennes de circulation maritime et continentale se sont diffusées et mêlées des influences culturelles venues du continent africain, de la péninsule arabique et de la péninsule indienne. Le développement du continent, du littoral et des archipels s'inscrit dans une double logique de fragmentation et d'ouverture au monde. Dans cette vaste aire géographique, le rôle de relais des îles et des axes de pénétration continentale à partir des ports est essentiel.

The identity and the original features of the Swahili seashore mainly proceed from the contact of African mainland and western indian Ocean. This coastal interface, stretching over 2000 kilometres from southern Somalia to northern Mozambique, is characterized by ancient traditions of trade movements through maritime areas and mainland as well, which spread and mixed different cultural influences originating from Africa and both Arabic and Indian peninsulas. The development of this area is linked to both fragmentation and opening dynamics. In such a wide area, the relaying function of islands and continental corridors from main harbours is very important. 
INDEX

Mots-clés : archipel, circulation, hinterland, littoral océanique, swahili

Keywords : archipelagos, circulation, hinterland, ocean shoreline, swahili

\section{AUTEUR}

\section{FRANÇOIS BART}

François Bart (fbart@ades.cnrs.fr) est professeur à l'Université de Bordeaux et membre de l'équipe CNRS/UMR 5185 ADES-DYMSET. Il a notamment publié :

- La montagne au cœur de l'Afrique orientale, Cahiers d'Outre-Mer, n 235, 2006, p. 307-322

- De Zanzibar au Kilimandjaro et à l'Afrique des Grands Lacs, Mondes et Cultures, tome LXVI, vol. 1, 2006, p. 485-492

- De l'île des girofliers à « l'île aux épices », In Bernardie N. (dir.), L'autre Zanzibar, Paris, Karthala, 2008, p. 69-84. 